part in the programme, of these $73 \%$ attended a screening clinic. Of these 64,104 were recalled for assessment. Of those recalled $81 \%$ did not have breast cancer, these women had a false-positive mammogram. The aim of this systematic review was to identify the psychological impact of false-positive screening mammograms and evidence for the effectiveness of interventions designed to reduce this.

Methods This was a systematic review using the principles of the Centre for Reviews and Dissemination in York.

All UK controlled studies and qualitative studies of women with a false-positive screening mammogram were included. The control group participants had normal mammograms. All psychological outcomes including returning for routine screening were permitted. Titles and abstracts were screened independently by two reviewers. Retrieved papers were reviewed and selected using the same independent process. Data were extracted by one reviewer and checked by another. Each included study was assessed for risk of bias.

Results The searches returned seven includable studies $(7 / 4,423)$. Those using disease specific measures found that there could be enduring distress that lasted up to three years; the level of distress was related to the degree of invasiveness of the assessment. At three years the relative risks were, further mammography 1.28 (95\% confidence interval 0.82 to 2.00), fine needle aspiration 1.80 (95\% confidence interval 1.17 to 2.77 ), biopsy 2.07 ( $95 \%$ confidence interval 1.22 to 3.52). There was also an enduring impact for those put on early recall 1.82 (95\% confidence interval 1.22 to 2.72), However, studies that used generic measures of anxiety and depression found no such impact up to three months after screening. The strongest evidence suggests that women with false-positive mammograms have an increased likelihood of failing to reattend for routine screening, relative risk 0.97 ( $95 \%$ confidence interval 0.96 to 0.98 ) compared to women with normal mammograms. No studies of women from different socio-economic or ethnic groups were found nor any published qualitative studies.

Conclusion Having a false-positive screening mammogram can cause breast cancer specific distress for up to three years. It is less likely there will be general anxiety or depression detectable at pathological levels. The degree of distress is related to the invasiveness of the assessment. Women with false-positive mammograms are less likely to return for routine assessment than those with normal ones.

\section{OP38 ACCOUNTING FOR NON-UPTAKE OF BOWEL CANCER SCREENING: A QUALITATIVE STUDY}

doi:10.1136/jech-2012-201753.038

C Palmer, M Thomas, R Raine, C Von Wagner. Department of Epidemiology and Public Health, UCL, London, England

Background Since being established in 2006, the English NHS Bowel Cancer Screening Programme (NHS BCSP) offers the faecal occult blood (FOB) test to adults aged $60-74$. This bowel cancer screening test is sent by post every two years to recipients who are required to provide six small samples of faeces from three bowel movements. The kit is returned by post to be tested for minute traces of blood that may be an early warning sign for the development of bowel cancer. Analysis shows that approximately $53 \%$ of people offered bowel cancer screening by FOB test take it up, and that uptake varies from $35 \%$ to $60 \%$ in the most and least deprived areas, respectively.

Methods We used a qualitative approach to explore views and experiences about bowel cancer screening in people who did not take part in the screening programme when invited to do so. We conducted 16 focus groups in London and South Yorkshire to generate extensive textual data, and analysed our data thematically using grounded theory techniques to explore and examine accounts of non-uptake. Previous research examining 'non-uptake', of this and other medical interventions has identified considerable challenges in terms of identifying and recruiting individuals who may be defined in this way. We therefore used a novel recruitment approach, working with the National BCSP Hubs to identify and recruit 'nonuptakers' of bowel cancer screening. We present the findings from our thematic analysis of 16 focus groups undertaken with people who did not participate in bowel cancer screening and explore the complexity of "non-uptakers" accounts.

Results \& Conclusion During preliminary analyses, we identified common accounts through which participants rationalised nonuptake and which present one or several barriers to undertaking bowel cancer screening. Participants rationalised their non-uptake of screening in a number of ways, for example by making reference to perceptions of low personal risk informed by 'feeling well' or having had no family history of bowel cancer. Others expressed sophisticated doubts about the benefits or value of the FOB test specifically, or in relation to diagnostic or treatment interventions for cancer more generally. Finally, the necessity to provide samples of faeces to complete the FOB test emerged repeatedly as a troubling aspect of the bowel cancer screening methodology offered by the English NHS BCSP.

\section{OP39 PROTECTION BY BCG AGAINST TUBERCULOSIS: SYSTEMATIC REVIEW AND META-REGRESSION ANALYSIS}

doi:10.1136/jech-2012-201753.039

'P Mangtani, ' $\mathrm{C}$ Ariti, ${ }^{1} \mathrm{~L}$ Rodrigues, ${ }^{2} \mathrm{~A}$ Abubakar, ${ }^{3} \mathrm{~L}$ Pimpin, ${ }^{1} \mathrm{P}$ Fine, ${ }^{1} \mathrm{P}$ Smith, ${ }^{4} \mathrm{~J}$ Sterne. ${ }^{1}$ Epidemiology and Population Health, LSHTM, London, UK; '2Department of Medicine, University of East Anglia, Norwich, UK; ${ }^{3}$ Department of Epidemiology, University of Cambridge, Cambridge, UK; ${ }^{4}$ Department of Epidemiology , University of Bristol, Bristol, UK

Setting Reasons for variation in BCG protection against tuberculosis are not well understood. We therefore estimated the protection provided by BCG vaccination against tuberculosis and examined reasons for variation in different settings.

Methods We systematically searched English and non-English articles in 10 databases from inception to May 2009 as well as sources such as Google Scholar and trial registers to October 2009. Search terms included tuberculosis, tubercle bacill*, M. tuberculosis, $M$. bovis, $M$. africanum, $M$. canetti, $M$. microti and $M$. tuberculosis. Terms for the intervention included BCG Vaccine, BCG, BCG Vacc*, BCG Imm*, bacillus calmette.

Two reviewers independently screened studies for inclusion. Data (including results and domains of study quality) were extracted by one reviewer and checked by another. Pooled analyses were based on random and fixed effects meta-analysis. Heterogeneity was quantified using tau-squared $\left(\tau^{2}\right)$ statistics. Random-effects meta-regression was used to investigate associations of study characteristics with intervention effect estimates.

Results Of 21,030 references we identified 21 trials reporting tuberculosis outcomes. Protection against tuberculosis disease was variable $\left(\tau^{2}=0.292\right)$. BCG efficacy was high at higher latitude or when BCG was given only to infants $(62 \%, 95 \%$ CI $48-72 \%)$ or to children after strict screening for tuberculin sensitivity $72 \% 95 \% \mathrm{CI}$ 60-81\%) Protection against meningeal and miliary tuberculosis combined was higher in infants $(90 \%)$ and children with stringent testing respectively $(92 \%)$. The observed protective effect of BCG did not differ by the strain of BCG vaccine used. Stringency of tuberculin testing to exclude those infected or sensitised to mycobacteria explained a good proportion of the observed variation with latitude $\left(\tau^{2}=0.292\right.$ in the null model, 0.100 in the bivariate model). In a multivariable meta-regression analysis there was strong evidence that infant vaccination or stringent prior tuberculin testing before BCG in childhood was associated with increased efficacy $(p=0.02)$ after taking into account latitude and a measure of study quality-diagnostic detection bias. 